\section{IDDF2021-ABS-0054 APPENDECTOMY INCREASES THE RISK OF COLORECTAL CANCER THROUGH CAUSING GUT MICROBIOTA DYSBIOSIS IN A LARGE POPULATION-BASED STUDY}

${ }^{1}$ Feiyu Shi* ${ }^{*}$ 'Gaixia Liu, ${ }^{2}$ Yufeng Lin, ${ }^{2}$ Cosmos liutao Guo, ${ }^{3}$ Jing Han, ${ }^{1} J u n j u n$ She. ${ }^{1}$ Department of General Surgery, The First Affiliated Hospital of Xi'an Jiaotong University, Xi'an, China; ${ }^{2}$ State Key Laboratory of Digestive Disease, Institute of Digestive Disease and Department of Medicine and Therapeutics, the Chinese University of Hong Kong, Hong Kong: ${ }^{3}$ Center for Gut Microbiome Research, Med-X Institute, The First Affiliated Hospital of Xi'an Jiao tong University, China

\subsection{6/gutjnl-2021-IDDF.24}

Background Appendix plays an important role in maintaining and modulating homeostasis and biodiversity of gut microbiome, by providing an ideal ecological niche for gut commensal bacteria and production of immunoglobulin A (IgA). Surgical removal of the appendix impacts the composition of gut microbial community and its homeostasis. We aim to elucidate the association between appendectomy and subsequent risk of colorectal cancer (CRC) development, and explore its relationship with gut microbiota dysbiosis.

Methods Two independent epidemiological studies involving 402,622 subjects were established to define the association between appendectomy and CRC risk longitudinally (IDDF2021-ABS-0054 Figure 1A, $\mathrm{n}=368,800$ ) and transversely (IDDF2021-ABS-0054 Figure 1B, $n=33,822$ ). Deep shotgun metagenomic sequencing was performed on fecal samples from an additional cohort (IDDF2021-ABS-0054 Figure 1C; 157 appendectomy cases and 157 normal controls without appendectomy).

Results Population-based longitudinal study showed an $18.6 \%$ increase in CRC risk among appendectomy cases compared with controls throughout 20 years follow-up (IDDF2021ABS-0054 Figure 2, Adjusted HR 1.19; 95\%CI 1.02-1.37). Consistently, a case-control study illustrated that appendectomy history was correlated with a higher risk of CRC
(Adjusted OR 1.83; 95\%CI 1.52-2.21). We evaluated the contribution of gut microbial composition change following appendectomy to the increased risk of CRC development. The altered microbial community profile in appendectomy cases (IDDF2021-ABS-0054 Figure 3) was observed with significant enrichment of 7 CRC-promoting bacteria (Bacteroides vulgatus, Bacteroides fragilis, Veillonella dispar, Prevotella ruminicola, Prevotella fucsa, Prevotella dentalis, Prevotella denticola); while 5 beneficial commensals (Blautia sp YL58, Enterococcus hirae, Lachnospiraceae bacterium Choco86, Collinsella aerofaciens, Blautia sp SC05B48) were depleted in appendectomy cases compared to the healthy controls(IDDF2021-ABS-0054 Figure 4). Furthermore, appendectomy altered the correlations among bacteria (IDDF2021ABS-0054 Figure 5A) and their functional pathways

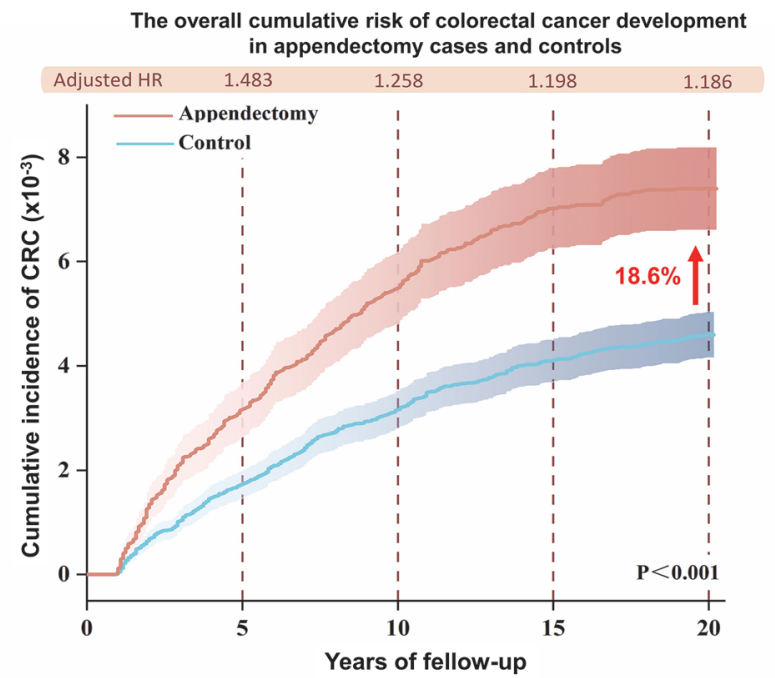

Abstract IDDF2021-ABS-0054 Figure 2

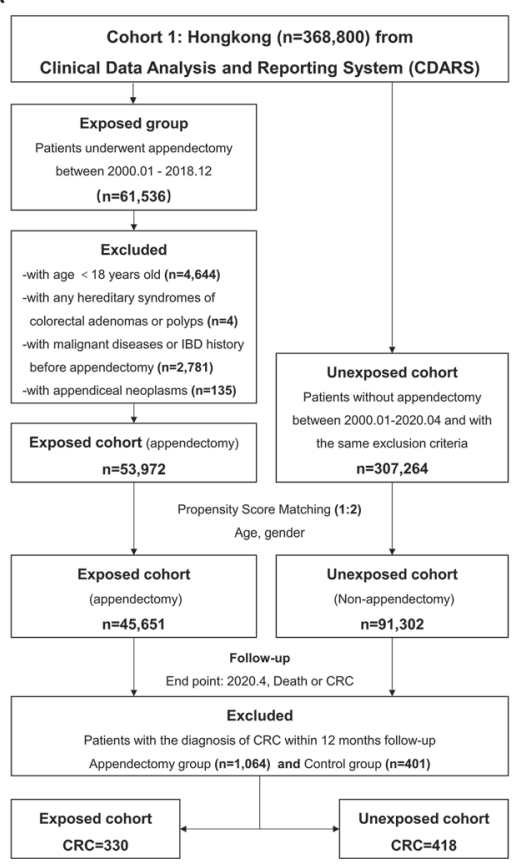

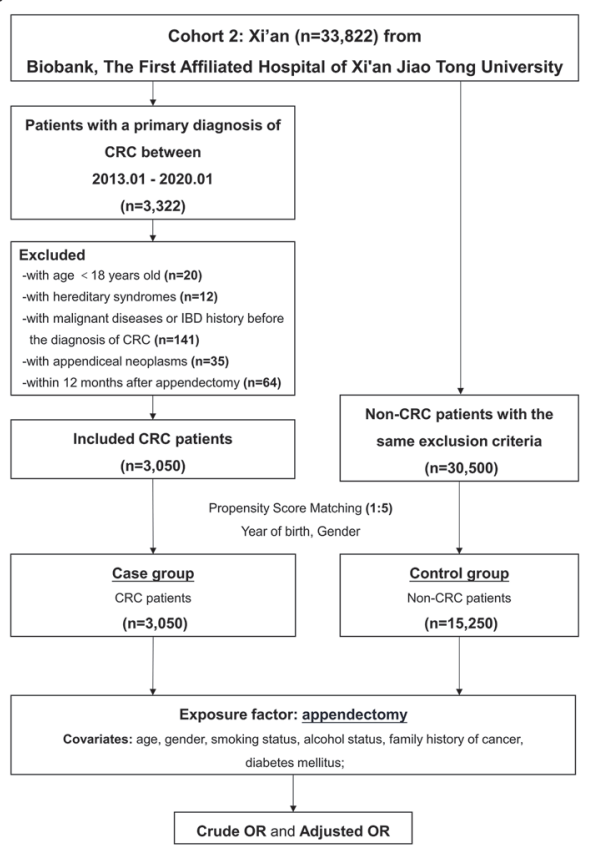

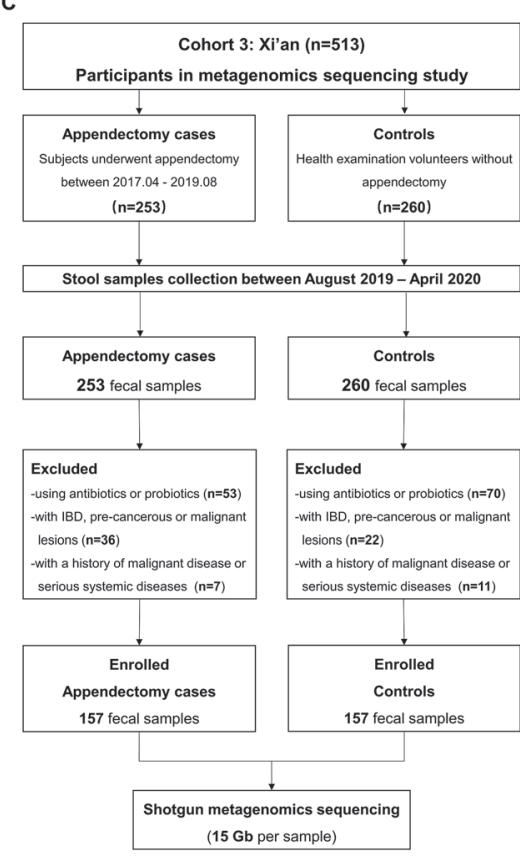


The microbial composition at phylum level in appendectomy and control groups
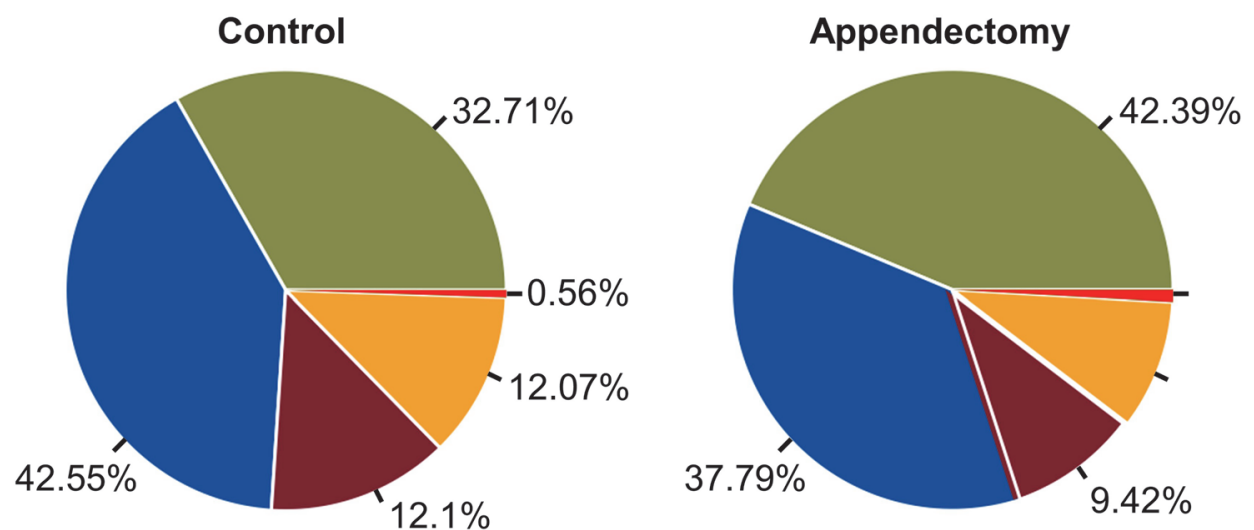

Firmicutes (TaxID1239)

Bacteroidetes (TaxID976) $\square$ Other

Actinobacteria (TaxID201174) $\square$ Proteobacteria (TaxID1224)

Alpha diversity and beta diversity at genus level in appendectomy and control groups
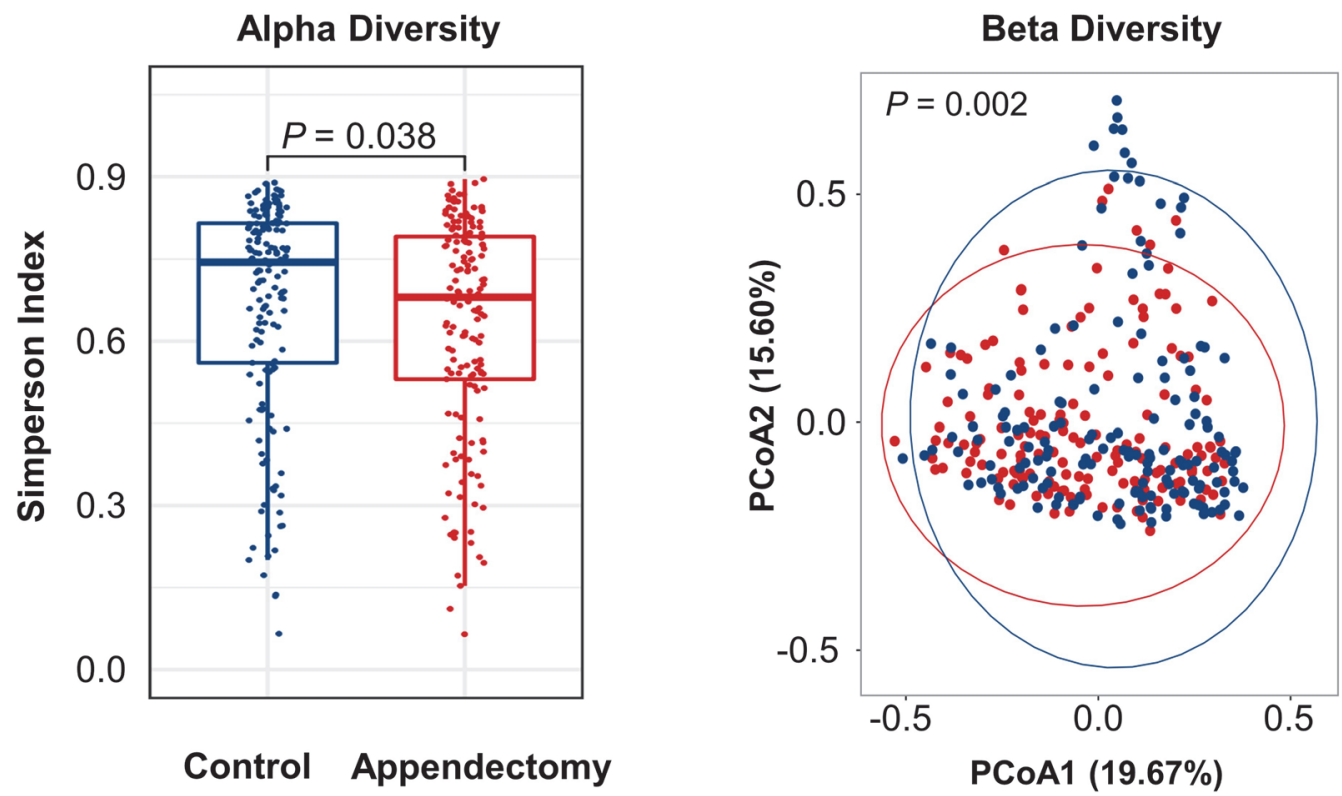

Abstract IDDF2021-ABS-0054 Figure 3 
(IDDF2021-ABS-0054 Figure 5B) to pro-inflammatory and pro-oncogenic gut microecological environment.

Conclusions Our population-based studies showed appendectomy significantly increases the risk of CRC development. Appendectomy-induced gut dysbiosis and related functional pathways contribute as least in part to the appendectomy-associated CRC risk.

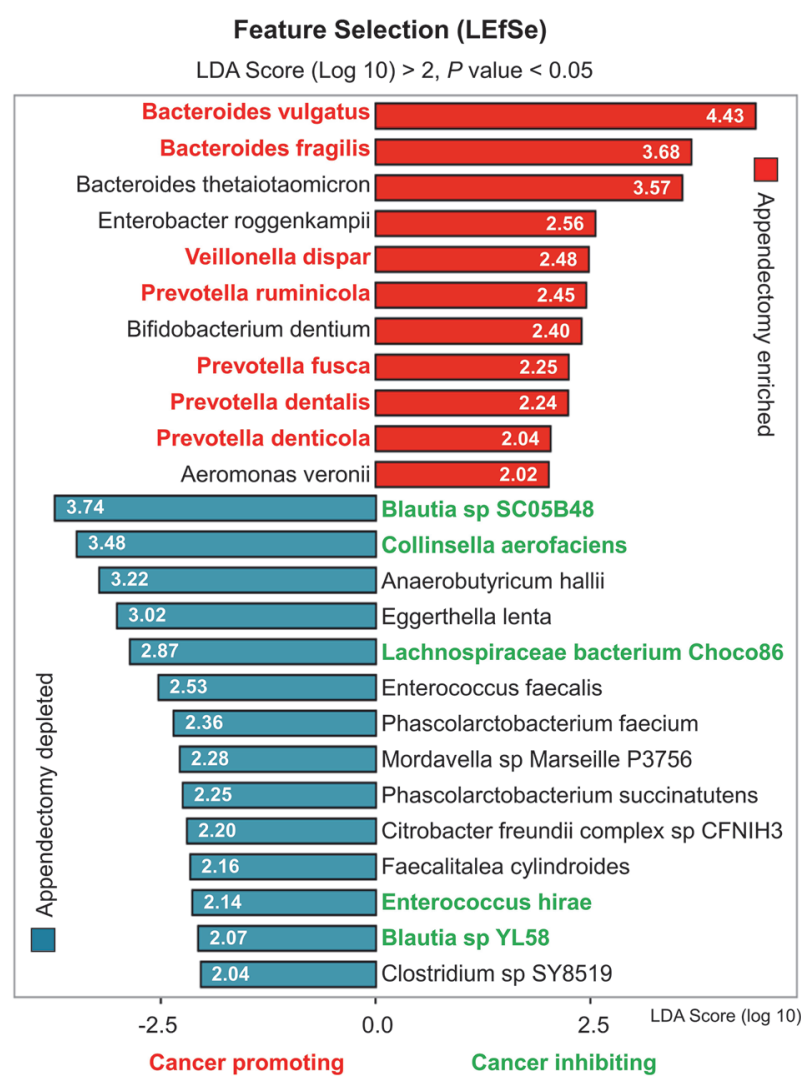

Abstract IDDF2021-ABS-0054 Figure 4

A

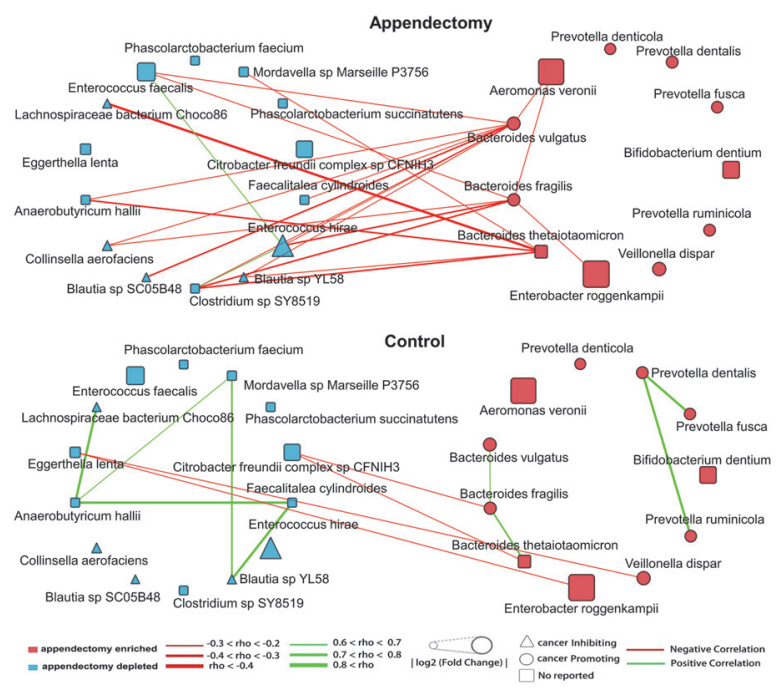

IDDF2021-ABS-0061

TM9SF4 AMELIORATES INFLAMMATORY
BOWEL DISEASE BY ALLEVIATING
INFLAMMATION AND ER STRESS IN
COLONIC EPITHELIAL CELLS AND
MACROPHAGES

${ }^{1}$ Mingxu Xie*, ${ }^{1}$ Hongyan Yu, ${ }^{1}$ Wing Hung Ko, ${ }^{2}$ Wing Yan Mak, 'Xiaogiang Yao. ${ }^{1}$ School of Biomedical Sciences, The Chinese University of Hong Kong, Hong Kong; ${ }^{2}$ Department of Medicine and Therapeutics, The Chinese University of Hong Kong, Hong Kong

\subsection{6/gutjnl-2021-IDDF.25}

Background Inflammatory bowel disease (IBD) is a result of excess inflammation and inappropriate immune response to environmental changes and the gut microbiota. The search of genome-wide association study (GWAS) database identified a remarkable correlation between TM9SF4 gene and IBD, with p-value $=6 \times 10^{10}$. In the previous studies, TM9SF4 was found to be highly expressed in macrophages and intestinal epithelial cells (IECs), promoting phagocytosis and innate immune response. Besides, TM9SF4 reduced cell stress to protect cells from apoptotic deaths. Therefore, it is likely that TM9SF4 might exert protective roles in IBD via its actions of reducing inflammation and alleviating ER stress in both macrophages and IECs.

Methods Expression levels of TM9SF4 were compared between actively inflamed and non-inflamed regions of colons from IBD patients by immunohistochemistry and western blotting. Dextran sulfate sodium (DSS)-induced colitis was established in wild-type (WT) and TM9SF4 knockout (KO) mice. Bone marrow transplantation was used to elucidate the contribution of myeloid cells and IECs in disease progression. Intestinal permeability, IECs stress and deaths, macrophage phagocytosis functions and polarization were measured with in vitro and in vivo techniques.

Results Decreased expression of TM9SF4 was observed in inflamed regions of colon tissues from patients with IBD. Patients with a higher Mayo score showed lower expression of TM9SF4 in their colons. In animal studies, knockout of TM9SF4 aggravated DSS-induced colitis, which could be attributed to TM9SF4 deficiency in bone marrow-derived hematopoietic cells as well as in colonic epithelial cells. For

B Functional prediction in appendectomy and control groups

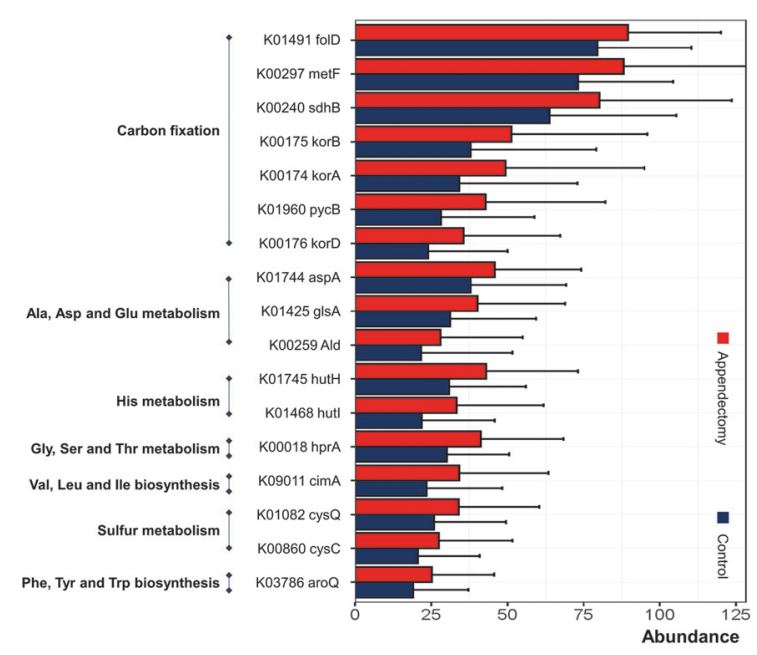

